\title{
Final scientific and technical report for grant DE-AI02-93ER40784: Fundamental Physics with Cold Neutrons (2007-2010)
}

\author{
M. Scott Dewey
}

February 7, 2013

The breadth of experiments performed using slow neutrons continues to increase worldwide. The accomplishments are a result of strong collaborations among national laboratories and university-based scientists and their students. There have been a growing number of notable results in fundamental neutron physics over the course of the last three-year grant period. A number of these experiments have been carried out on the beamlines at NIST or with the participation of NIST researchers. The list below gives a brief summary of some of these results as well as some of the cooperative endeavors during the last grant period.

- The data run for the measurement of neutron spin rotation in ${ }^{4} \mathrm{He}$ was completed in June of 2008. The analysis produced a value on the rotation angle per unit length of $d \phi / d z=(+1.7 \pm 9.2) \times 10^{-7} \mathrm{rad} / \mathrm{m}$, which contributes to the constraints on nucleonnucleon couplings. A paper detailing this measurement will be published in Physical Review C (R). The collaboration consists of Gettysburg, Georgia State, Indiana, Joint Institute for Nuclear Research - Dubna, NIST, North Carolina Central, Washington, and George Washington.

- The second data run on an experiment to make a precision measurement of the branching ratio and energy spectrum of the radiative decay of the free neutron was completed in November of 2009. The data analysis is in progress, and the collaboration is optimistic that they will reach the goal of a $1 \%$ precision. A detailed article describing the first measurement was published in Physical Review C in 2009. The work is performed in a collaboration among Arizona State, Maryland, Michigan, NIST, Tulane, and Sussex.

- In early December 2009, the aCORN apparatus was shipped from the Indiana Center for the Exploration of Energy and Matter (CEEM) to NIST, where it was installed on the NG-6 beamline. The experiment is designed to determine the electron-antineutrino correlation by measuring an asymmetry rather than the shape of the proton spectrum. The apparatus is undergoing testing with cold neutrons, and the preliminary data 
is being analyzed. The collaboration members include Depauw, Hamilton, Indiana, NIST, Tulane and Sussex.

- The analysis of the data from the emiT experiment is largely complete. This experiment will set the best limit on the time-reversal violating $D$ coefficient in neutron beta-decay. A manuscript is currently in preparation for submission to Physical Review Letters. The collaboration includes researchers from the Berkeley/LBNL, Hamilton, Michigan, NIST, North Carolina, Tulane, and Washington/CENPA.

- A collaboration involving NIST, Tennessee, and Tulane is working on the determination of absolute neutron fluence with the Alpha-Gamma instrument. The period of data acquisition is nearing completion. The apparatus has demonstrated the ability to accurately count neutrons at the $0.1 \%$ level and should result in an improvement to the NIST beam lifetime measurement. This novel technique also provides a direct way of determining some standard neutron cross sections, such as ${ }^{6} \mathrm{Li}$ and ${ }^{10} \mathrm{~B}$.

- The ultracold neutron lifetime apparatus underwent a major upgrade to a larger magnet which should ultimately allow a measurement of the neutron lifetime at the few second level. The collaborators have successfully completed testing of this magnet and assembled the apparatus on the NG-6u beamline. The acquisition of preliminary data is now in progress. The experiment is a joint effort of NIST and North Carolina State University.

- Recently it was shown that the maximum observed longitudinal relaxation times (T1) of polarized ${ }^{3} \mathrm{He}$ cells can be used to set limits on short range spin-dependent forces. Members of the polarization groups at NIST and Indiana realized better limits could be obtained using the transverse relaxation time, T2. A manuscript that sets limits based on both existing long T2 values and a proof-of-principle experiment was accepted for publication in Physical Review D (R).

- A precise measurement of the $n^{3} \mathrm{He}$ incoherent scattering length using neutron interferometry was successful, resulting in the first use of polarized gas with a neutron interferometer and the first interferometry-based measurement of the spin dependence of the scattering length. The results were published in Physical Review Letters in 2009. The work was done as a collaboration among Indiana, Tulane, NIST, and North Carolina - Wilmington.

- The data analysis for the NPDGamma experiment is complete, and a manuscript has been accepted for publication in PRC. The result of the gamma asymmetry is $A_{\gamma}=$ $(-1.8 \pm 2.1) \times 10^{-7}$. A ${ }^{3}$ He polarization of $58 \%$ was obtained in a neutron polarizer that was used in the experiment. The experiment ran at Los Alamos National Laboratory, and recently the apparatus was disassembled and moved to the Spallation Neutron Source where it will be the first experiment to acquire data on the Fundamental Physics Neutron Beamline. 
Recently completed Ph.D.s

\begin{tabular}{llll}
\hline Name & Affiliation & Ph.D. & Thesis topic \\
\hline Christopher Bass & Indiana & 2008 & $\mathrm{n}-{ }^{4}$ He spin rotation \\
Robert Cooper & Michigan & 2008 & radiative neutron decay \\
Carroll Trull & Tulane & 2008 & emiT and aCORN \\
Venera Zhumabekova & Al-Farabi Kazakh National & 2008 & neutron spin rotation \\
Michael Huber & Tulane & 2009 & n- ${ }^{3}$ He scattering length \\
Da Luo & Indiana & 2009 & neutron spin rotation \\
Chris O'Shaughnessy & N. Carolina State & 2010 & UCN neutron lifetime \\
\hline
\end{tabular}

Table 1: Graduate student participation in the research program of the Neutron Interactions and Dosimetry Group. Seven graduate students received their Ph.D. on experiments performed at NIST during the last grant period.

- In June of 2009, NIST hosted the second Summer School on Fundamental Neutron Physics (http://www.ncnr.nist.gov/summerschool/ss09/index_fundphys.html). The school had 37 registered students from 18 universities and twelve speakers and organizers. Seven of the participants came from outside the US (Germany, Canada, Britain, and Mexico). It received financial support from DoE-Nuclear Physics, IUCF/LENS, NSF, NIST, Tulane, and ORNL/Tennessee.

- Table 1 lists the seven Ph.D. students who completed their theses on experiments performed within our group. 